\title{
The emission-line spectrum of the UV deficient quasar Ton 34: evidence of shock excitation?
}

\author{
L. Binette $\mathrm{e}^{1,2}$ and Y. Krongold ${ }^{2}$ \\ 1 Département de Physique, de Génie Physique et d'Optique, Université Laval, Québec, QC, G1K 7P4, Canada \\ 2 Instituto de Astronomía, UNAM, Ap. 70-264, 04510 México, DF, México
}

Received 12 July 2007 / Accepted 20 September 2007

ABSTRACT

\begin{abstract}
Context. Emission lines in quasars are believed to originate from a photoionized plasma. There are, however, some emission features that appear to be collisionally excited, such as the Fe II multiplet bands. Shortward of Ly $\alpha$, there are also a few permitted lines of species from low to intermediate ionization.

Aims. Ton $34\left(z_{q}=1.928\right)$ exhibits the steepest far-UV continuum decline known $\left(F_{v} \propto v^{-5.3}\right)$ shortward of $1050 \AA$ A. This object also emits unusually strong low to intermediate-excitation permitted lines shortward of the Lyman limit.

Methods. Using archive spectra of Ton 34 from HST, IUE, and Palomar, we measured the fluxes of all the lines present in the spectra and compared their relative intensities with those observed in composite quasar spectra.

Results. Our analysis reveals unusual strengths with respect to Ly $\alpha$ of the following low to intermediate-excitation permitted lines: O II+O III (835 ̊), N III+O III (686-703 Å), and N III+N IV (765 Å). We compared the observed line spectrum with both photoionization and shock models.

Conclusions. Photoionization cannot reproduce the strengths of these far-UV lines. Shocks with $V_{\mathrm{s}} \simeq 100 \mathrm{~km} \mathrm{~s}^{-1}$ turn out to be extremely efficient emitters of these lines and are favored as an excitation mechanism.
\end{abstract}

Key words. line: identification - line: formation - atomic processes - galaxies: quasars: emission lines galaxies: quasars: individual: Ton 34

\section{Introduction}

In this work, we analyze the emission lines of an unusual quasar, Ton 34 , which is alternatively named PG $1017+280$ or $\mathrm{J} 1019+2745$ with redshift $z_{q}=1.928$. It is severely deficient in ionizing photons, since its spectral energy distribution (SED) shows a remarkable steepening of the continuum in the restframe far-UV, shortward of $1100 \AA$ (Binette \& Krongold 2007, hereafter BK07; Binette et al. 2007). If the far-UV is fitted by a power law $\left(F_{v} \propto v^{+\alpha}\right)$, the index ${ }^{1}$ is as steep as $v^{-5.3}$. BK07 suggest that the extreme-UV flux might undergo a recovery shortward of $450 \AA$

While the near-UV emission-line spectrum appears to be "normal", the far-UV spectrum shows low to intermediate ionization species with unusual strengths. Using the UV SED constructed by BK07 from archive data, we will quantify this statement and present photoionization and shock models for comparison. The aim is to understand how the extreme deficiency of ionizing photons in Ton 34 might be impacting the emission-line spectrum.

The emission-line spectrum of quasar and Seyfert I galaxies is generally believed to originate from gas photoionized by a nuclear UV source. State-of-the-art photoionization models of the broad emission line region (BELR), such as those developed by Baldwin et al. (1995) and dubbed "locally optimally emitting clouds" (LOC) models, can successfully reproduce most of the emission lines observed in quasars. A grid of these models

\footnotetext{
1 Among the 77 quasars whose far-UV indices could be measured by Telfer et al. (2002), there were only 3 objects with a continuum steeper than $v^{-3}$
}

can be found in Korista et al. (1997, hereafter KO97) and more recently in Casebeer et al. (2006 and references therein). There are, however, a few exceptions to the success of pure photoionization. In particular, photoionization models require microturbulences in order to reproduce the shape and intensity of the Fe II UV-band (Baldwin et al. 2004). A possible alternative is that the region producing Fe II is collisionally ionized, as proposed by Grandi (1981, 1982), Joly (1987), Véron-Cetty et al. (2004, 2006), and Joly et al. (2007). In this work, we present evidence that photoionization might not be sustainable in the case of some of the far-UV permitted lines reported in this paper.

\section{The UV emission-line spectrum of Ton 34}

Below we summarize the procedure used by BK07 to derive the UV SED of Ton 34.

\subsection{Description of the archival data}

The current work is based on four archival or bibliographical sources. The 760-1120 $\AA$ spectral segment is provided by the dataset Y2IE0A0AT from the HST-FOS archives (grating $\mathrm{G} 270 \mathrm{H}$ ). To cover the extreme UV region, we borrowed from the IUE archives. The long wavelength segment (LWP) is from Tripp, Bechtold \& Green (1994) and corresponds to the dataset LW0P5708. Fluxes longward of $3000 \AA$ (observerframe) were severely affected by reflected sunlight or moonlight (Lanzetta et al. 1993) and have been discarded. The shorter wavelength IUE segment (SWP) was extracted directly from the archives and corresponds to the dataset SWP28188. To cover the 
SED behavior longward of the HST segment, we adopted the published optical spectra of Sargent et al. (1988), which were taken at the Palomar $5.08 \mathrm{~m}$ Hale Telescope. Both optical spectra lacked absolute flux calibration, although the authors observed standard stars, which allowed them to provide a relative calibration.

\subsection{Matching the different SED segments}

We statistically corrected the UV spectral segments for the cumulated absorption caused by unresolved Ly $\alpha$ forest lines, which are responsible for the so-called far-UV "Lyman valley" (Møller \& Jakobsen 1990). For that purpose, we adopted the mean ${ }^{2}$ transmission function for $z_{q}=2$ published by Zheng et al. (1997). We also applied a Galactic reddening correction assuming the Cardelli et al. (1989) extinction curve corresponding to $R_{V}=3.1$ and $E_{B-V}=0.13$. The latter value corresponds to the mean extinction inferred from the $100 \mu$ maps of Schlegel et al. (1998) near Ton 34. The blue and red arm segments have been scaled to overlap smoothly with the HST-FOS segment. Both the LWP and SWP segments were multiplied by a factor 0.75 . This scaling was necessary so that the LWP segment superimposes the HST-FOS spectrum as closely as possible. Continuum variability is a possible explanation for this continuum difference, since the IUE and HST observations were made in different years. Finally, all the spectral segments were shifted to rest-frame wavelengths, and $F_{\lambda}$ was multiplied by $1+z_{q}$. The IUE spectra have been re-binned by grouping $n$ pixels together (SWP with $n=5$ and LWP with $n=3$ ) to improve the limited $S / N$. The LWP and HST-FOS spectra overlap significantly in spectral coverage. Both datasets taken nine year apart confirm the unusual steepness of the UV break in Ton 34 .

\subsection{Model of the ionizing SED of Ton 34}

Shortward of $1100 \AA$, the continuum of Ton 34 undergoes a sharp fall off (see Fig. 2 in BK07), which BK07 model as dust absorption by nanodiamond grains. This resulted in a deep and broad absorption trough that fits the observed continuum reasonably well. In our photoionization calculations presented below in Sect. 3.2.1, we experiment with two ionizing SEDs. The first is the intrinsic "unabsorbed" SED, which is assumed to be a power law of index +0.1 followed by a roll-over centered on $640 \AA$ that extends up to the X-ray domain. Beyond $2 \mathrm{keV}$, SED II behaves as a power law of index -1.0 , yielding an $\alpha_{\mathrm{OX}}$ of -1.45 . This SED is shown in Fig. 1 and, as in the work of BK07, it is labeled Model II. The second SED used in photoionization calculations is the dust-absorbed version of the same SED, which fits the $o b$ served UV continuum of Ton 34 between 400 and $1550 \AA$ (labeled Model IV in Fig. 1). Shortward of $200 \AA$ and longward of $2000 \AA$, the two distributions are the same. This is because nanodiamond dust absorbs radiation over a relatively narrow domain as compared to other grain compositions. In Fig. 2, we present the continuum subtracted spectrum of Ton 34 , that is, the residual between the observed Ton 34 SED and our continuum fit represented by Model IV.

2 This correction is statistical in nature, as it relies on the average behavior with redshift of the spatial density of intervening absorbers. It cannot be used to correct small portions of the continuum, which may be coincident with a "clear patch" or an over-density in the Ly $\alpha$ forest. These inhomogeneities may generate spurious narrow features that should not be attributed to genuine emission lines.

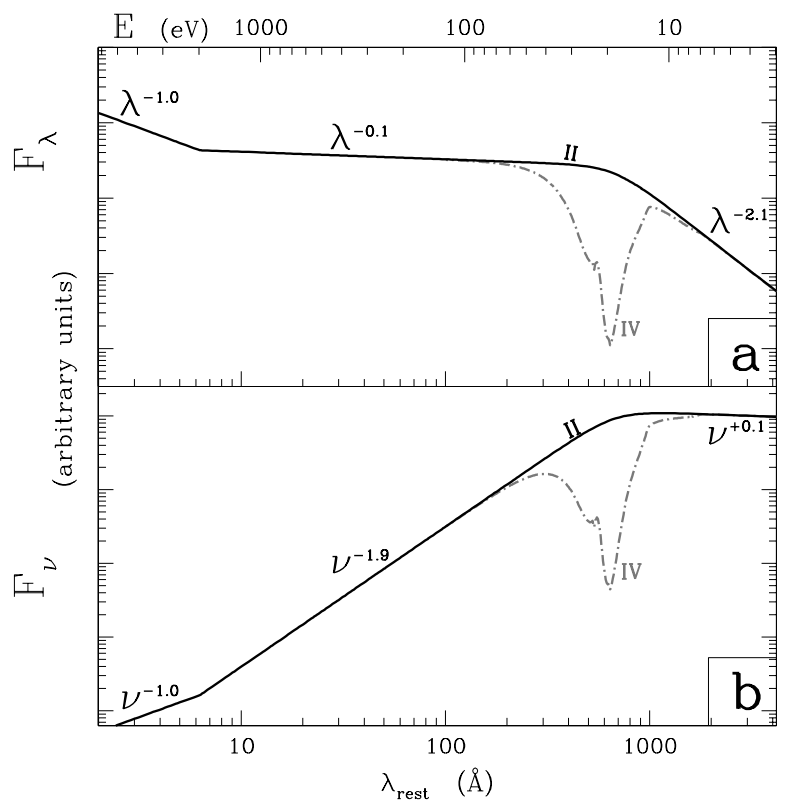

Fig. 1. Log-log plot of the input spectral energy distributions used in our photoionization calculations discussed in Sect.3.2.1. These ionizing SEDs are labeled II and IV in either $F_{\lambda}$ (panel a)) or $F_{v}$ (panel b)) and are given as a function of wavelength (bottom axis) or photon energy (top axis). The distribution labeled II (solid line) is the assumed intrinsic SED, while that labeled IV is the transmitted flux (dash-dotted line) assuming nanodiamond dust extinction (see BK07). Model IV is a fit of the UV continuum of Ton 34 between 400 and $1550 \AA$.

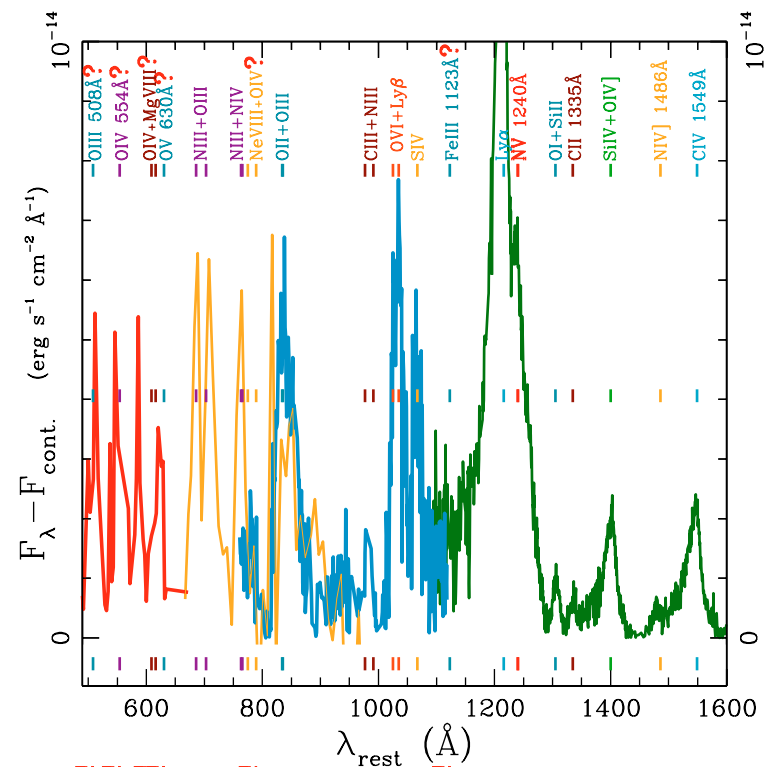

Fig. 2. Residuals of the spectral energy distribution of Ton 34 after subtracting our absorbed continuum Model IV from BK07. The different spectral segments have been color-coded as follows, SWP: red, LWP orange, HST-FOS: blue, and Palomar: dark green. Color-coded fiducial marks indicate the position of observed or expected (labeled with symbol "?") emission lines. Measurements of line intensities and upper limits are given in Table 1.

\subsection{Extraction of line fluxes and upper limits}

The procedure for measuring the flux of the lines was the following: we first fit a Gaussian to each observed line in the spectra. For several lines, a narrow component was required, so we added a second (narrow) Gaussian. In addition, the lines by C IV $\lambda 1549$, 
Si IV $\lambda 1400$, and Ly $\alpha$ show a clear asymmetry in the line profile, with a blue shoulder (see Fig. 2). For these lines, we included a third, broader Gaussian. The FWHM of the broad component spans from $\sim 3600$ to $5300 \mathrm{~km} \mathrm{~s}^{-1}$. It is interesting to note that the O II+O III complex at around $835 \AA$ has a significant and strong red shoulder extending up to $\sim 850 \AA$, which is observed in both the IUE-LWP and HST-FOS spectra (see Fig. 2). We could not find any positive identification of this shoulder with any line from a different ion/transition, so we considered this feature as part of the $\mathrm{O}$ II $+\mathrm{O}$ III emission.

The measured line fluxes extracted from Fig. 2, as well as upper limits of other permitted lines, are listed with respect to Ly $\alpha=100$ in Col. 5 of Table 1. Note that we give the total flux under the profile, that is, the integrated flux from all the Gaussian components required to fit each emission line. A consistency check was carried out, which showed that the line fluxes measured over the original spectra or the continuum subtracted spectra were indistinguishable from each other.

In Col. 5 of Table 1, we show our error estimates, which we evaluated at a $1 \sigma$ significance level. We assumed an $S / N$ of 25 for most lines, except for N III+N IV and N III+O III, where we assume a, $S / N$ of $\simeq 10$. The line upper limits in Table 1 correspond to a significance of $2 \sigma$. As for the continuum, we estimate the errors to be $\simeq 10 \%$.

Of all the emission features that we measure in the far-UV, three line systems stand out by their strengths with respect to the composite spectra: the O II+O III lines at $835 \AA$, the N III+O III lines at $686-703 \AA$, and the N III+N IV lines at $765 \AA$.

Many weaker features in the IUE spectrum appear to lie where other permitted lines of comparable excitation might be expected, such as O III $\lambda 508 \AA$, O IV $\lambda 554 \AA$, O v $\lambda 630 \AA$, and O IV $\lambda 609 \AA$. A few of these have been reported before in other quasars (Reimers et al. 1998; Laor et al. 1995) or in composite AGN spectra (Zheng et al. 1997; Telfer et al. 2002; Scott et al. 2004). However, these line systems appear too narrow in the IUE spectra compared to typical BELR line profiles (see the profile comparison of Fig. 3). They lack a broad component at their base. Given the limited $S / N$ of the IUE spectrum at the farUV end, we consider it probable that these lines are spurious features instead. For this reason, we consider these emission-like features as upper limits rather than real detections. The symbol "?" denotes these unconfirmed lines in our various figures.

We find little evidence of the high excitation Ne VIII line at $775 \AA$ reported by Telfer et al. (2002) and Scott et al. (2004) in their respective composite spectrum, and we favor the identification of O IV $\lambda 789 \AA$ instead. Because the line spectrum of Ton 34 has unusually low excitation as shown below in Sect. 3.1, we do not believe that the high excitation lines of $\mathrm{MgX}$ and $\mathrm{Ne}$ VIII (listed in Table 1) are present at a detectable level.

\subsection{Originality and limitations of the data}

As can be gathered from Fig. 2, the strongest emission features in the far-UV coincide with the position of lines observed or expected in quasar spectra (Sect. 3.1). However, the limited quality of the data and the possible coincidence of absorbers at inconvenient spectral positions prevent us from deriving incontrovertible conclusions. In the case of the narrower features (O III $\lambda 508 \AA$, O IV $\lambda 554 \AA$, O V $\lambda 630 \AA$, and O IV $\lambda 609 \AA$ A), better quality data is required to confirm or discard their presence, as discussed in Sect. 2.4. Clearly, new observations are needed in all wave bands down to the X-rays. In what follows, we take the data at face

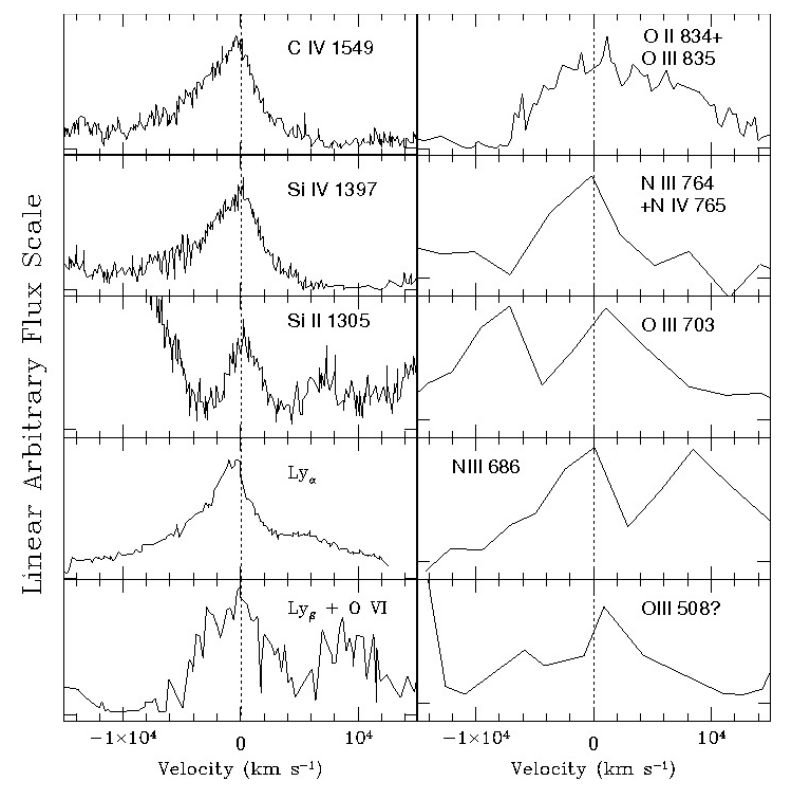

Fig. 3. Emission lines extracted from the Ton 34 spectrum plotted in velocity space. The flux scale is arbitrary for each inset. Left panels: nearUV permitted lines, right panel: far-UV permitted lines. Overall, the lines are all consistent with the rest-frame system of Ton 34. Differences in the position of the lines, on the right panel, may be due to absorption by intergalactic gas. The narrow line of O III at $508 \AA$ (bottom right panel) is severely affected by intergalactic absorption, and better data would be required to confirm its presence. The same applies to the other lines shown as upper limits in Table 1.

value and present photoionization and shock models that attempt to reproduce the far-UV lines.

\section{Modeling the line spectrum}

\subsection{Line ratio comparison with composite quasar spectra}

We now quantify to what degree the emission lines differ in Ton 34 from the "average" quasar. To achieve this, we list the line ratios characterizing the radio-loud (Col.3) and radio-quiet (Col.4) composite spectra of Telfer et al. (2002) in Table 1. Comparison between Ton 34 and these two sets of ratios requires some caution, since significant line ratio variations exist among quasars. For instance, Telfer et al. (2002) report that the RMS deviation of line fluxes between the different quasars amounts to as much as $50-70 \%$ for the strong lines of C IV $\lambda 1549$, O VI $\lambda 1035$, and $\operatorname{Ly} \alpha$. Hence, intrinsic differences of less than a factor two between the composites and Ton 34 should not be considered significant.

To facilitate the comparison of Ton 34 with the two composites, we plot their line ratios in Fig. 4. Inspection of the Table 1 or Fig. 4 reveals that the commonly strong BELR lines of C IV, $\mathrm{N} \mathrm{V}$, and $\mathrm{O}$ VI are all present in Ton 34. As a result, the apparent sharp turndown of the ionizing UV in the range 650-912 $\AA$ is not radically affecting the high excitation emission lines. In particular, the O VI $\lambda 1035$ line is quite strong, although not as much as in the two composites. The C IV is substantially weaker, by more than a factor of six in Ton 34 with respect to the radio-quiet composite. Also, the line system C III+N III near $980 \AA$ is noticeably weaker, although the flux in this line is difficult to measure accurately due to the uncertainties introduced by the sharp continuum bent and the many $\operatorname{Ly} \alpha$ forest lines.

In the far-UV, we note that the intensity of the O II+O III and $\mathrm{N}$ III+O III systems in Ton 34 are a factor of $\sim 14$ and 18 brighter, respectively, than in the RLQ composite. There is also evidence 
Table 1. Comparison of Ton 34 with composite SEDs and with models.

\begin{tabular}{|c|c|c|c|c|c|c|c|c|}
\hline \multicolumn{2}{|c|}{ Lines } & \multicolumn{3}{|c|}{ Observations } & \multicolumn{3}{|c|}{ Photoionization $^{a}$} & \multirow{2}{*}{$\begin{array}{c}\text { Shocks }^{a, b} \\
100^{d} \mathrm{~km} \mathrm{~s}^{-1}\end{array}$} \\
\hline Species & $\lambda(\AA)$ & RLQ & RQQ & Ton 34 & $\mathrm{KO} 97^{c}$ & $\operatorname{SED~II~}^{d}$ & SED IV $^{d}$ & \\
\hline (1) & (2) & (3) & (4) & (5) & (6) & (7) & (8) & (9) \\
\hline O III & 508 & $<1$ & $<1$ & $\leq 5.1$ & $?$ & $10^{-2.4}$ & 0.02 & 8.7 \\
\hline O IV & 554 & $<1$ & $<1$ & $\leq 4.1$ & $?$ & 0.04 & 0.13 & 5.7 \\
\hline Ne VIII & 575 & $<1$ & $2.1^{e}$ & - & $?$ & $?$ & $?$ & $?$ \\
\hline He I & 601 & - & - & $\leq 0.5$ & $?$ & 2.3 & 0.9 & 2.9 \\
\hline $\mathrm{O}$ IV+Mg X & 609,617 & $<1$ & $<1$ & $<4.7$ & $0.04+1.07$ & $0.03+?$ & $0.1+?$ & $2.0+?$ \\
\hline $\mathrm{OV}$ & 630 & $<1$ & $<1$ & $\leq 2.8$ & 0.6 & 0.13 & 0.25 & 0.06 \\
\hline N III+O III & 686,703 & $1.5^{e}$ & $<1$ & $27 \pm 4.9$ & $?$ & $0.05+0.35$ & $0.05+0.78$ & $6.8+22$ \\
\hline N III+N IV & 764,765 & $<1$ & $<1$ & $\mathbf{1 1} \pm 1.4$ & $0.02+0.08$ & $0.04+0.14$ & $0.04+0.04$ & $2.2+3.2$ \\
\hline $\mathrm{Ne}$ VIII+O IV & 775,789 & 4.7 & 5.7 & $\leq 2.2$ & $2.4+1.1$ & $?+0.67$ & $?+1.6$ & $?+5.4$ \\
\hline $\mathrm{O}$ II+O III & 834,835 & 1.2 & 1.5 & $\mathbf{2 1} \pm 0.32$ & $?+0.5$ & $0.04+1.4$ & $0.04+2.4$ & $48+23$ \\
\hline $\mathrm{C}$ II+N II & 906,912 & - & - & $\leq 2.7$ & $?$ & $<10^{-2}$ & $<10^{-2}$ & $22+4.1$ \\
\hline $\mathrm{C}$ III+N III & 977,991 & 8.1 & 11.7 & $5.4 \pm 0.64$ & $2.9+0.7$ & $4.9+0.8$ & $3.9+0.5$ & $13+7.0$ \\
\hline $\mathrm{Ly} \beta+\mathrm{O}$ VI & 1025,1035 & 19.1 & 18.1 & $14 \pm 1.75$ & $1.1+20$ & $0.37+1.5$ & $0.36+21.5$ & $2.5+10^{-5}$ \\
\hline C II & 1037 & - & - & blended $^{f}$ & $?$ & 0.02 & 0.02 & 4.1 \\
\hline $\mathrm{N}$ II+He II & 1084 & 5.6 & 5.5 & - & $0.07+0.6$ & $0.01+?$ & $0.01+?$ & $4.3+?$ \\
\hline S IV & 1067 & $<1$ & $<1$ & $5.7 \pm 0.23$ & $?$ & 0.97 & 0.95 & 1.6 \\
\hline Fe III & 1123 & 0.28 & 2.2 & - & 0.01 & 0.01 & $<10^{-2}$ & 0.07 \\
\hline C III & 1176 & 0.44 & 0.43 & - & 0.4 & 4.8 & 4.9 & 1.7 \\
\hline S III+Si III & 1194,1207 & 1.5 & 0.47 & blended $^{f}$ & $0.04+1.0$ & $0.02+0.11$ & $0.02+0.06$ & $0.5+5.0$ \\
\hline $\operatorname{Ly} \alpha$ & 1216 & 100 & 100 & $100^{g} \pm 10.2$ & 100 & 100 & 100 & 100 \\
\hline $\mathrm{OV}$ & 1218 & - & - & blended $^{f}$ & 5.3 & 2.7 & 12.8 & $10^{-4}$ \\
\hline $\mathrm{NV}$ & 1240 & 22.0 & 17.5 & $4.4 \pm 0.2$ & 3.0 & 1.5 & 6.7 & 0.03 \\
\hline Si II & 1262 & 0.27 & 0.41 & - & 0.08 & 0.03 & 0.05 & 1.3 \\
\hline $\mathrm{O}$ I+Si II & 1302,1305 & 1.9 & 2.5 & $2.1 \pm 0.24$ & $0.07+0.03$ & $10^{-5.6}+0.01$ & $10^{-5.0}+0.02$ & $10^{-6.9}+0.15$ \\
\hline C II & 1335 & 0.35 & 1.0 & $1.4 \pm 0.13$ & 0.7 & 0.63 & 0.7 & 44 \\
\hline Si IV+O IV] & 1397,1402 & 8.6 & 11.9 & $9.2 \pm 0.8$ & $3.5+2.4$ & $2.9+1.8$ & $1.1+0.8$ & $3.9+0.9$ \\
\hline N IV] & 1486 & 2.8 & 0.6 & $1.3 \pm 0.2$ & 2.6 & 3.5 & 4.5 & 0.3 \\
\hline C IV & 1549 & 52 & 38 & $12 \pm 1.2$ & 59 & 44 & 61.4 & 15 \\
\hline He II & 1640 & 1.2 & 1 & $0.7 \pm ?$ & 3.0 & 4.0 & 9.5 & 2.1 \\
\hline O III] & 1664 & 2.3 & 0.7 & - & 7.8 & 8.1 & 6.3 & 0.7 \\
\hline
\end{tabular}

${ }^{a}$ Some observational entries in Cols. (3)-(5) correspond to the sum of two different lines. For the corresponding models in Cols. (6)-(9), we list each line intensity separately using a + symbol as separator.

${ }^{b}$ Redward of $1700 \AA$ (down to the infrared), the $100 \mathrm{~km} \mathrm{~s}^{-1}$ shock does not generate any strong lines. For completeness, the only other lines of significant brightness are Si III $1896 \AA$, Ca II $3969 \AA$, and Ca II $3934 \AA$, whose intensities reach 3.6\%, 1.5\%, and 3\% of the intensity of Ly $\alpha$, respectively. As for the (optical and UV) Fe II multiplet line systems, we cannot say since they are not considered by MAPPINGS Ic. Shortward of $400 \AA$, we expect the He II lines to be strong, with He II $304 \AA$ A reaching $80 \%$ of Ly $\alpha$.

${ }^{c}$ A crude model that approximates the optimally locally emitting BELR model described by Baldwin et al. (1995). Each line's peak emissivity was extracted from the grid AGN4 of photoionization calculations published by Korista et al. (1997)

${ }^{d}$ These three models were computed with MAPPINGS Ic assuming an initial density $n_{\mathrm{H}}^{0}$ of $4 \times 10^{9} \mathrm{~cm}^{-3}$ and solar metallicities. The ionization parameter is 0.04 for the two photoionized models and zero for the shock model. At these densities, the Ly $\alpha$ luminosities per unit area of photoionized or shocked gas are $3.2 \times 10^{7}, 4.4 \times 10^{7}$ and $5.5 \times 10^{5} \mathrm{erg} \mathrm{cm}^{-2} \mathrm{~s}^{-1}$ for models shown in Cols. (7)-(9), respectively. These would scale approximately in proportion to $n_{\mathrm{H}}^{0}$.

${ }^{e}$ Measurement by one of us (YK) using the composite spectra lent by R. Telfer.

${ }^{f}$ The strong neighboring lines of $\operatorname{Ly} \alpha$ or O VI makes the determination of a meaningful upper limit impossible.

${ }^{g}$ The Ly $\alpha$ flux in Ton 34 is measured to be $6.9 \times 10^{-13} \mathrm{erg} \mathrm{cm}^{-2} \mathrm{~s}^{-1}$ corresponding to an equivalent width of $57 \AA$.

of significant emission of N III and/or N IV at 764 and $765 \AA$, which are not detected in the composite spectra either.

\subsection{Photoionization vs. shock excitation}

The line spectrum of Ton 34 show peculiarities that deserve further analysis, in particular, O II+O III (835 ̊), N III+O III lines
(686-703 $\AA$ ), and N III+N IV (765 $\AA$ ), which are measured with unusual strengths with respect to $\operatorname{Ly} \alpha$. Are these emission features necessarily genuine lines? One possibility is that extinction resonances, unaccounted for in the extinction curve used to model the deep continuum trough (BK07), may induce features that looked like broad emission lines. Another possibility is that Ly $\alpha$ absorbers at intervening redshifts might generate 


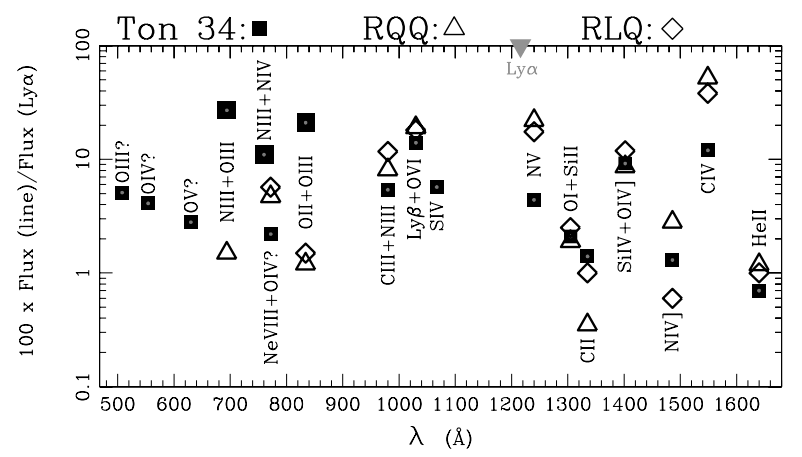

Fig. 4. Line flux ratios renormalized to $\operatorname{Ly} \alpha=100$ of different species as a function of line wavelength $(\AA)$. These were extracted from the spectrum of Ton 34 (filled squares) and from the radio-loud (crosses) and radio-quiet (open lozenge) composite spectra of Telfer et al. (2002). Only ratios that have a counterpart in Ton 34 are shown. Larger squares correspond to ratios for which the difference between Ton 34 and the composites exceeds a factor 10 (also shown in bold face in Col. 5 of Table 1). The symbol "?" denotes upper limits of unconfirmed lines in Ton 34. The gray filled triangle indicates the position of $\operatorname{Ly} \alpha$.

spurious emission features by bracketing narrow continuum regions. Although we cannot rule out either possibility with the current data, both appear unlikely to us, on the grounds that the strongest emission features coincide quite well with the position of plausible atomic transitions (see Fig. 2). The two strongest line systems of O II+O III (835 $\AA$ ) and N III+O III (686-703 $\AA$ ) have previously been reported in the RLQ composite, although at a much reduced flux level. We thus pursue our analysis under the assumption that the observed features are real and consist of low to intermediate-excitation permitted lines.

\subsubsection{Photoionization calculations}

Can photoionization account for the strength of the far-UV permitted lines? We first establish a comparison with published BELR models and then evaluate the impact of a strongly absorbed ionizing continuum.

Baldwin et al. (1995) show that by integrating line fluxes over a wide range in gas density $n_{\mathrm{H}}$ and impinging ionizing flux $\varphi_{\mathrm{H}}$, one obtains a much improved fit to quasar line spectra. Such models were dubbed "locally optimally emitting clouds" (LOC). Baldwin et al. (1995) also show that by preferentially selecting the optimal slab density and impinging flux for each individual line, one can derive a line spectrum comparable (within a factor two) to that of a true LOC model. To derive an approximate LOC model, we proceeded as follows. From the grid of photoionization models published by Korista et al. (1997; hereafter KO97), we extracted the highest equivalent width found within the plane $\varphi_{\mathrm{H}}$ vs. $n_{\mathrm{H}}$, for each line of interest. The particular grid that we selected was labeled AGN $4^{3}$. It assumes solar abundances and an SED that was defined by KO97, which peaks at $22 \mathrm{eV}$. It is the closest to our SED II with a $18.5 \mathrm{eV}$ turnover (Fig. 1; see also Haro-Corzo et al. 2007).

The line ratios from this approximated LOC model are shown in Col. 6 of Table 1. Unfortunately, the $\mathrm{N}$ III+O III line system $(\lambda \lambda 686-703 \AA)$ was not part of the AGN4 grid, nor was the O II $\lambda 834 \AA$ line. On the other hand, the N III+N IV system at $765 \AA$ and the $\mathrm{O}$ III line at $835 \AA$ were. The N III+N IV system

3 The selected grid AGN4 comprises over a 100 lines and is available at http://www . pa.uky . edu/ korista/grids/grids.html is significantly weaker than observed, while the $\lambda 835 \AA$ O III line is predicted an order of magnitude weaker than the observed O II+O III system. As we consider unlikely that the $\lambda 834 \AA$ O II line (absent from the AGN4 grid) is stronger than O III, we conclude that photoionization would have difficulty in fitting this system. Hence, even locally optimally emitting clouds would not be able to account for the intensities of at least some of the farUV lines observed in Ton 34.

Could the peculiar shape of the Ton 34 SED be responsible for the unusual strengths of some far-UV lines? Out of curiosity, we calculated photoionization models with the multipurpose code MAPPINGS Ic (Ferruit et al. 1997; Binette et al. 1989), using SED II to compare with the absorbed SED IV, characterized by the deep trough. We assumed solar metallicities (Anders \& Grevesse $1989)$ and a gas density of $4 \times 10^{9} \mathrm{~cm}^{-3}$. The ionization parame$\operatorname{ter}^{4}$ was varied until a maximum in the $\mathrm{O}$ III $] / \mathrm{H} \beta(\lambda 1663 / \lambda 4861)$ ratio was found, which occurred at $U=0.04$. The models were truncated at a depth where $\mathrm{H}$ is $10 \%$ ionized. These calculations with $U=0.04$ using either SED II or IV (both plotted in Fig. 1) are reported in Cols. 7 and 8 of Table 1, respectively. Because there are fewer soft ionizing photons in SED IV, we find that the mean energy of the photoelectrons is twice as high as the one given by SED II. This must result in a hotter plasma and therefore in stronger collisionally excited lines. A comparison of the calculated ratios between the two models and with Ton 34 (Col. 5) reveals that, although many metal lines in Col. 8 (SED IV) are often stronger than in Col. 7 (SED II), the deep UV trough does not result in a sufficient increase in the strengths of either the O III+N III lines at $683,703 \AA$ or of the O II+O III lines at $835 \AA$. In conclusion, photoionization predicts far-UV line intensities that are much too weak in comparison with our measurements. Furthermore, making drastic changes in the shape of the ionizing continuum does not alter this conclusion.

\subsubsection{Cooling shock calculations}

In view of the difficulties producing strong permitted lines of O II, O III, and N III in the case of pure photoionization, we are lead to consider whether collisional ionization might not be more appropriate.

To investigate this possibility, we used MAPPINGS Ic to calculate a sequence of steady-state plane-parallel shock models with a preshock density of $4 \times 10^{9} \mathrm{~cm}^{-3}$, again assuming solar metallicities. The postshock temperatures of the different models covered the range $1.0 \times 10^{5}-8 \times 10^{5} \mathrm{~K}$, corresponding to shock velocities of 75 to $235 \mathrm{~km} \mathrm{~s}^{-1}$. The pre-ionization state of the shocked gas was determined self-consistently by an iterative scheme, using the ionizing radiation produced within the cooling shock that propagates upstream (Dopita et al. 1984). The time evolution of the electron and ion temperatures was followed separately until they equalized, making use of the equilibration timescale as defined by Spitzer (1962). Most of the far-UV resonance lines are emitted downstream in layers of densities in the range $10^{10.6}-10^{11.3} \mathrm{~cm}^{-3}$, well below the densities of $10^{16}$ where collisional de-excitation would become a concern for many resonance lines. The elapsed time for the shocked gas to cool to temperatures of $8500 \mathrm{~K}$ is about $10 \mathrm{~s}$. The adiabatic cooling and recombination of the plasma was followed in time until the ionized fraction reached $\leq 2 \%$. Because the integrated columns of the different ions are modest in shocks, line opacities turn out

\footnotetext{
4 We use the customary definition of the ionization parameter $U=$ $\varphi_{\mathrm{H}} / c n_{\mathrm{H}}$, which is the ratio of the density of ionizing photons impinging on the slab $\varphi_{\mathrm{H}} c$ to the $\mathrm{H}$ density at the face of the slab $n_{\mathrm{H}}^{0}$.
} 


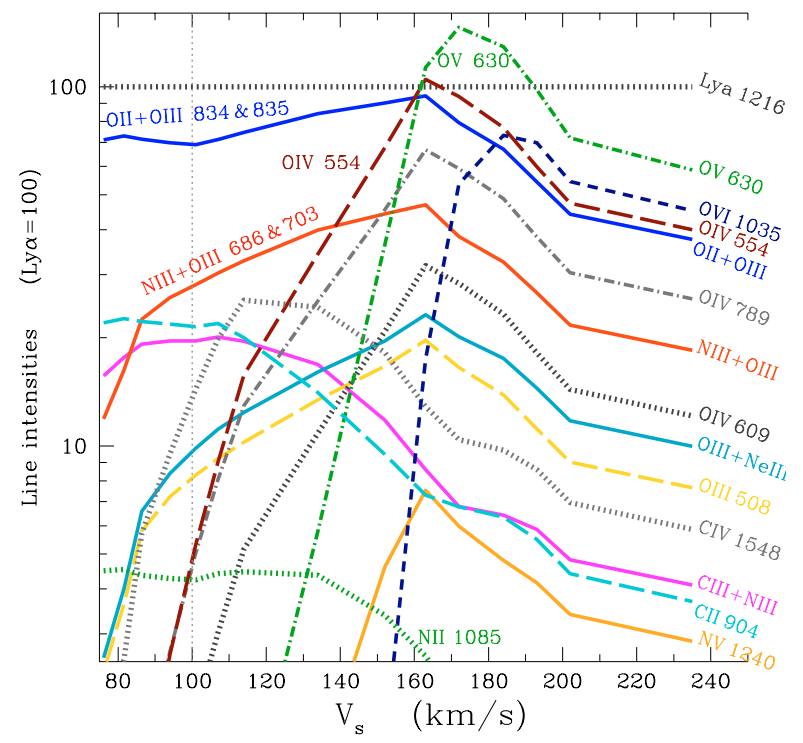

Fig. 5. Line intensities from high-density cooling shocks renormalized to $\operatorname{Ly} \alpha=100$ as a function of shock velocity. Solar metallicities have been assumed. A vertical dashed line denotes the velocity of the shock model reproduced in Table 1.

to be negligible compared to those of photoionized slabs. For instance, the line-center opacity of C III $\lambda 977$ and C IV $\lambda 1549$ are 20 and 1 , respectively, for a $100 \mathrm{~km} \mathrm{~s}^{-1}$ shock, compared to $10^{5.3}$ and $10^{4.9}$ for the photoionization model of Col. 8 .

The intensities of representative far-UV lines are shown in Fig. 5 as a function of shock velocity. The calculations show that shocks with gas densities appropriate to the BELR are very efficient in producing strong lines of O II+O III ( $\lambda 835 \AA)$ and of N III+O III $(\lambda \lambda 686-703 \AA)$, which reach $71 \%$ and $29 \%$ of Ly $\alpha$, respectively. We also computed the intensities of many other far-UV lines that might be observable in future observations. Some high-excitation lines such as O IV $\lambda 554 \AA$, O IV $\lambda 789 \AA$, and O v $\lambda 630 \AA$, become intense for shock velocities exceeding $120 \mathrm{~km} \mathrm{~s}^{-1}$. By comparing the observed upper limits for these lines in Table 1 with the computed intensities of O III $\lambda 835 \AA$ or O III $\lambda 703 \AA$, we find that velocities on the order of $90-130 \mathrm{~km} \mathrm{~s}^{-1}$ produce line intensities compatible with the estimated line ratios ${ }^{5}$. To be definite, we adopted the velocity of $100 \mathrm{~km} \mathrm{~s}^{-1}$ for the case model ${ }^{6}$ presented in Col. 9 of Table 1.

Shock models by themselves predict far-UV line intensities that are too strong with respect to $\operatorname{Ly} \alpha$ (compare Cols. 9 and 5), creating a reverse situation to that of photoionization (Sect. 3.2.1). We are therefore lead to propose a mixed model, in which we ascribe only a fraction of the luminosity of Ly $\alpha$ to shock excitation and the complementary fraction to photoionization. In this mixed model, photoionization would be responsible for the emission of the strong near-UV (i.e. classical) lines, while shocks would be contributing about a third of $\operatorname{Ly} \alpha$ and (proportionally) all of the far-UV resonance lines shortward of the Lyman limit.

\footnotetext{
5 While the measurements for O IV $\lambda 554 \AA$ and O V $\lambda 630 \AA$ formally represent only upper limits, it remains possible that the intensities of these lines are somewhat larger than evaluated given the limited $S / N$ of the IUE-SWP spectrum and the possible presence of many intergalactic absorption lines (this would imply higher shock velocities).

${ }^{6}$ For completeness, Table 1 includes all the lines that the shock model predicts to be stronger than $2 \%$ of Ly $\alpha$ within the reported domain of 400-1700 ̊.
}

The preshock density $n_{\mathrm{H}}^{0}$ may be significantly higher than assumed above. We find similar line ratios for preshock densities up to 100 times higher. The luminosity per unit area of the shock model in this case exceeds that of the photoionization models presented in Cols. 7 and 8 (see footnote $a$ in Table 1). Our code includes three-body recombination of $\mathrm{H}$, but not the process of stimulated emission, which prevents us from going beyond a preshock density of $10^{11.6} \mathrm{~cm}^{-3}$. Beyond this limit, we expect Ly $\alpha$ to be the first line to thermalize, which would further enhance the strengths of the metal lines with respect to Ly $\alpha$.

In summary, the far-UV lines observed in Ton 34 shortward of the Lyman limit are characterized by a much lower excitation energy than the near-UV lines. For this reason, collisional excitation (through shocks) at temperatures significantly higher than typically provided by photoionization is strongly favored. Calculations with MAPPINGS Ic show that such a temperature regime is ensured when shock excitation of moderate $V_{\mathrm{s}}$ takes place. These shocks would not only account for the far-UV lines, but may also contribute significantly to the Fe II multiplet lines that have been proposed as resulting from mechanical heating by Joly et al. (2007, and references therein).

Acknowledgements. This work was supported by the CONACyT grants J-50296 and J-49594, and the UNAM PAPIIT grant IN118905. Diethild Starkmeth helped us with proofreading.

\section{References}

Anders, E., \& Grevesse, N. 1989, Geochim. Cosmochim. Acta. 53, 197 Baldwin, J., Ferland, G., Korista, K., \& Verner, D. 1995, ApJ, 455, L119 Baldwin, J. A., Ferland, G. J., Korista, K. T., Hamann, F., \& LaCluyzé, A. 2004, ApJ, 615, 610

Binette, L., \& Krongold, Y. 2007, A\&A, in press (BK07)

Binette, L., Prieto, A., Szusziewicz, E., \& Zheng, W. 1989, ApJ, 343, 135

Binette, Haro-Corzo, S., L., Krongold, Y., \& Andersen, A. C. 2007, in Proc. of the nuclear region, host galaxy and environment of active galaxies, Huatulco, México, 18-20 April 2007, ed. E. Benítez, I. CrúzGonzalez, \& Y. Krongold, Rev. Mex. Astron. Astrofis. Conf. Ser., in press, [arXiv:astro-ph/0706.2006]

Cardelli, J. A., Clayton, G. C., \& Mathis, J. S. 1989, ApJ, 345, 245

Casebeer, D. A., Leighly, K. M., \& Baron, E. 2006, ApJ, 637, 157

Dopita, M. A., Binette, L., \& Tuohy, I. R. 1984, ApJ, 282, 142

Ferruit, P., Binette, L., Sutherland, R. S., \& Pécontal, E. 1997, A\&A, 322, 73

Grandi, S. A. 1981, ApJ, 251, 451

Grandi, S. A. 1982, ApJ, 255, 25

Haro-Corzo, S. A. R., Binette, L., Krongold, Y., et al. 2007, ApJ, 662, 145

Joly, M. 1987, A\&A, 184, 33

Joly, M., Véron-Cetty, M., \& Véron, P. 2007, in Proc. of the nuclear region, host galaxy and environment of active galaxies, Huatulco, México, 18-20 April 2007, ed. E. Benítez, I. Crúz-Gonzalez, \& Y. Krongold, Rev. Mex. Astron. Astrofis. Conf. Ser., in press

Korista, K., Baldwin, J., Ferland, G., \& Verner, D. 1997, ApJS, 108, 401 (KO97) Lanzetta, K. M., Turnshek, D. A., \& Sandoval, J. 1993, ApJS, 84, 109

Laor, A., Bahcall, J. N., Jannuzi, B. T., Schneider, D. P., \& Green, R. F. 1995, ApJS, 99, 1

Møller, P., \& Jakobsen, P. 1990, A\&A, 228, 299

Reimers, D., Köhler, S., Hagen, H.-J., \& Wisotzki, L. 1998, ESA SP-413: Ultraviolet Astrophysics Beyond the IUE Final Archive, 579

Sargent, W. L. W., Boksenberg, A., \& Steidel, C. C. 1988, ApJS, 68, 539

Schlegel, D. J., Finkbeiner, D. P., \& Davis, M. 1998, ApJ, 500, 525

Scott, J., Kriss, G. A., Brotherton, M. S., et al. 2004, ApJ, 615, 135

Spitzer, L. 1962, Physics of fully ionized gases (New York: Interscience)

Telfer, R. C., Zheng, W., Kriss, G. A., \& Davidsen, A. F. 2002, ApJ, 565, 773

Tripp, T. M., Bechtold, J., \& Green, R. F. 1994, ApJ, 433, 533

Véron-Cetty, M.-P., Joly, M., \& Véron, P. 2004, A\&A, 417, 515

Véron-Cetty, M.-P., Joly, M., Véron, P., et al. 2006, A\&A, 451, 851

Zheng, W., Kriss, G. A., Telfer, R. C., Grimes, J. P., \& Davidsen, A. F. 1997, ApJ, 475, 469 